\title{
STIDIO DI ALCUNE ESPLOSIONI SUBACQUEE NEL GOLFO DI TRIESTE
}

\author{
Cario Morelut
}

1.-- Premessa. Nei primi anni unesesivi alla fine della guerra, cono sali rastrellati nel porto di Triese e nelle vicinanze numerosi re-iduali bellici, che venisano poi falli e-plodere nei dintorni dellat cillat, o in male. Le esplosioni in terra, sia per la maggiore distanzal, rome per il lallo di arvenire alla superfieie del suolo, non sono state registralle in generale dalla stazione Si-mica di Tricse. Tranne in ruilehe caleo ed in modo imperentibile. Le e-plo-ioni in matre hamno dato invere molto epeso regi-tazioni cospiene. e lalsolta anzi sono -late arvertite dalla popolarione con tulle le caralleri-liche di un piecolo erremoto lorale.

Valesal lat penat quindi di effelluturne uno -ludio. sia per il partlieolare mezo-ambiente in au ese arevano origine e si propaga-

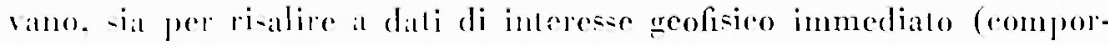
lamento elatico degli trati superfieiali-ini nei dintorni della Stazione: in particolare. dromocrone locali, aneora seonociute o più ge-

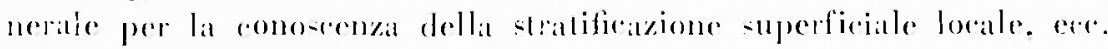

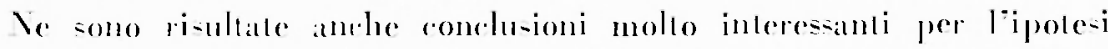
-ulle rau-e dei microsi-mi.

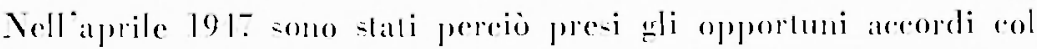
locale Comando Ratacellatori Bombe o Mine e col Comando del Por10. I quali ateordarono lautorizazione di awomplagnare in mare $i$ -ommozzalori allo -ropo di ceeguire le mi-urazioni necesaric per la deleminazione dei punti di soppio.

2. - Esplosioni - Dati sperimentali. I dali relativi alle coplosoni di mine. che lamno fornito il materiale sperimentale poito a base della precente riecrea, risultano dalfia seguente tabella, in ordine di di-tanzal dallit Slazione regi-lratriee (che nomalmente $i$ Trie-te.

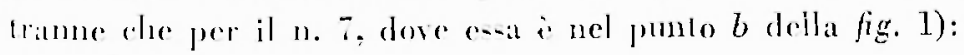




\begin{tabular}{|c|c|c|c|c|c|c|}
\hline$\dot{\vec{E}}$ & Dala & 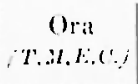 & $\begin{array}{l}\text { Profomulita } \\
\qquad(m)\end{array}$ & $\begin{array}{c}\text { Dislitllzil } \\
\qquad(m)\end{array}$ & Iximul & Vו11. \\
\hline 1 & 20.1 .119 .1$. & (9) $19^{m}$ & 2.2 .11 & 310100 & 3180101 & in nllitr. \\
\hline$\because 2$ & $28.1 .191 \%$ & 937 & 201.5 & 3025 & 32615 & $"$ \\
\hline 3 & 30.1 .19 .1 - & 1) 11 & 2010.11 & 3.1110 & $32.3 \quad 30$ & $"$ \\
\hline 1 & $28.1 .191 \%$ & 659 & 19.5 & 3850 & 320115 & » \\
\hline 5 & $311.1 .1^{11.17}$ & 10168 & $\mid 9,0$ & 16.511 & 3.310 & $"$ \\
\hline 0 & 2.5 .1917 & 1) 118 & 19.11 & 51,30 & $321 \quad 30$ & $"$ \\
\hline 7 & 23.3 .1418 & 1500 & - & 615 & 317 & $\begin{array}{l}\text { Save di } \\
\text { Si-l litllit }\end{array}$ \\
\hline 8 & 25.1 .1917 & 021 & 9.2 & "isiin & 32930 & in miare \\
\hline 9 & 23.3 .19 .18 & 1,110 & - & 16235 & 326001 & $\begin{array}{l}\text { lias di } \\
\text { Si-1iatllit }\end{array}$ \\
\hline 10 & 5.8 .14 .30 & $1: 01$ & - & 102015 & $3 \geq 6 \quad 1111$ & $n$ \\
\hline
\end{tabular}

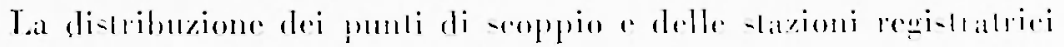
risulta dialla tig. 1 .

sono necesatrie le seguenti ossertasion pre lo mine in malre:

a) le mine epplose elano hombe aldatlate a mine. da 1000 k di trilolo:

b) la prima eplo-ione is slata simulantea di dare mine:

c) le mine renivimo appograiale - 11 londo del matre:

d) il pumo di scoppio 2 stalo delerminalo medianla il meloslo

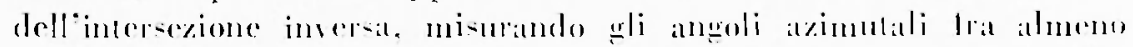

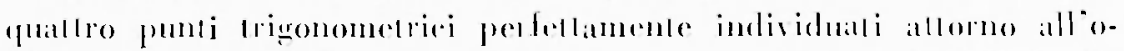
rizzonle: riporlalo il punlo sullat arlat, ne sono sali raleolalli la die stanza e laximnl rispetlo alla Slaxione. sopma indieali.

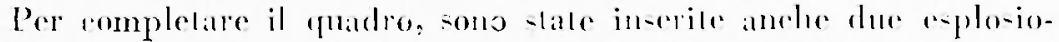

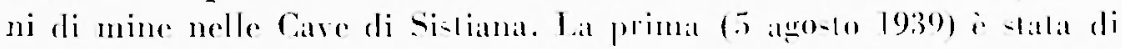

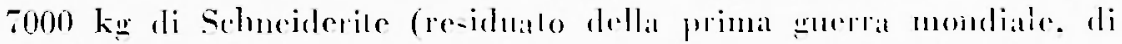

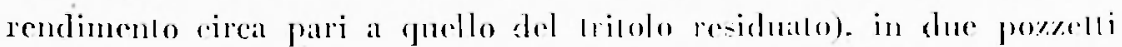
conligui con $\mathrm{kgr}+200$ e 2800 riepellisimenle. alla profondita di t5

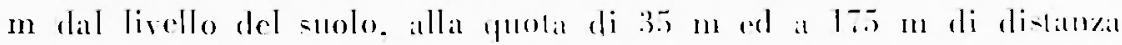
dal mare. Il tragilto delle onde sismiehe i qua-i sompledamente soltomarino per arrivare alla Stazione Si-mina di 'Tlie-le a 16285 m.

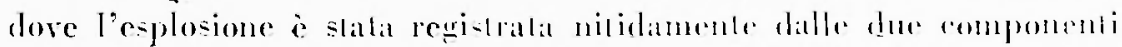
orizz. Alfani a registrazione folografiea fquelle dei Wieckerl a regi-tr. meceanica sono andale distrulle durunte il lombatramento di Triesere del 10 gingno 194, che colpi in pieno anche larchivio sismogrammit.

La seconda esplosione alle Cave di Si-liana (23 mamo 19.18) $i=l a-$

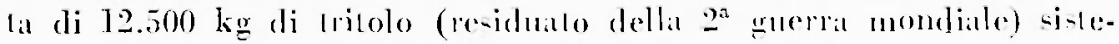




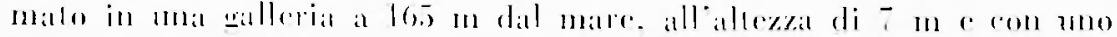

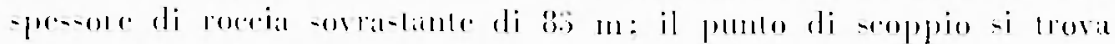

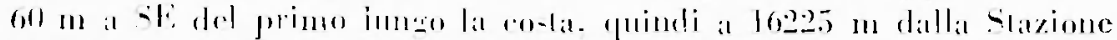

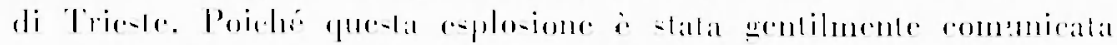

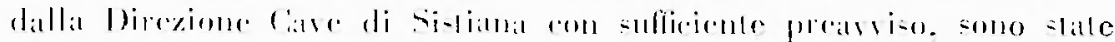

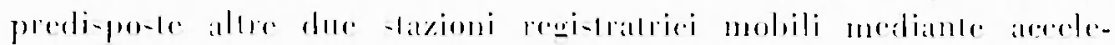

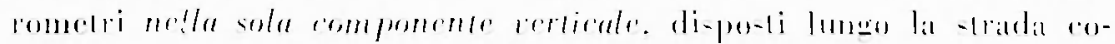

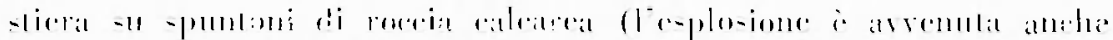

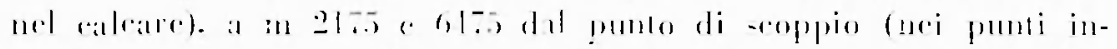

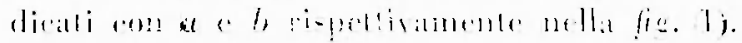

11 primo i 11 in aceelerometro Geiger. matricola $n$. 33t, co-truito datla Lethman- Viehet-di Amburqo, di proprietit della Fabbrical Macehine $s$. Indrea, Trie-te re golabile -neli insrandimenti di 3 . 6. 12 - 21 volte. Data la piecoleza dell ingrandimento manimo raggiungibile. \& tato portato alla di-tanza minima con-entita dalle mi-ure di -icureza: ma neppure co-i la regi-ltazione è -lata apprezzallile.

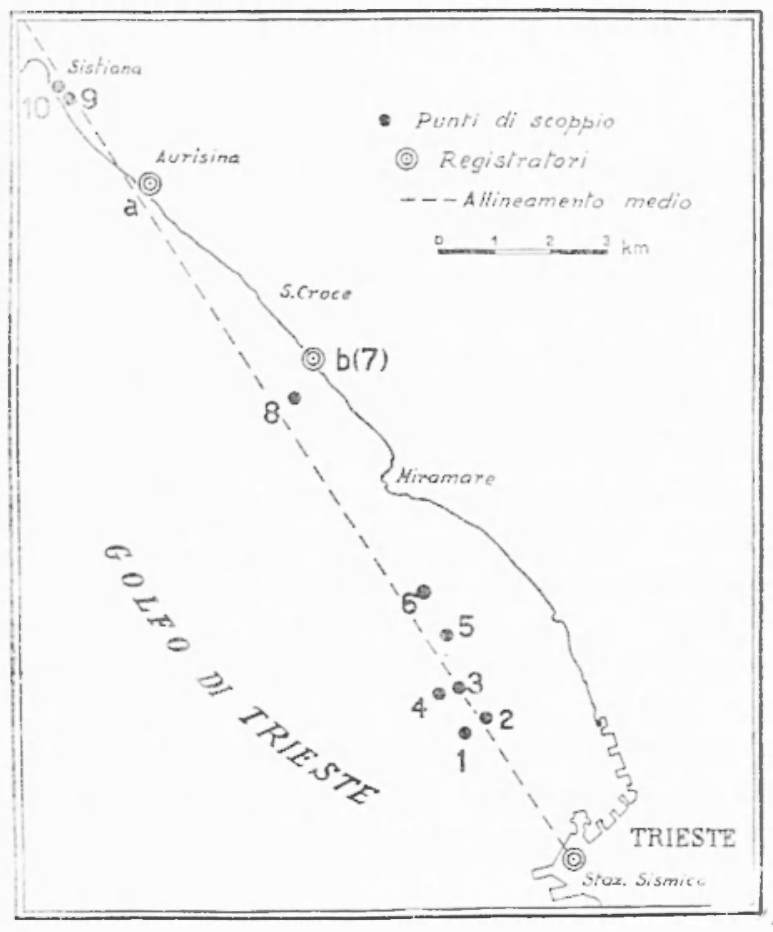

II -enomla i int

l i... 1

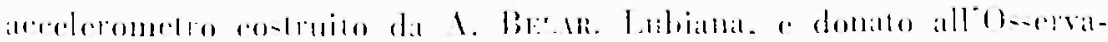

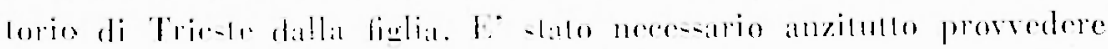

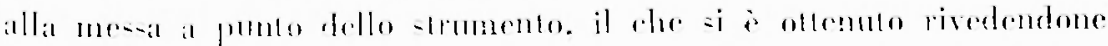

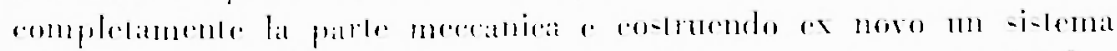

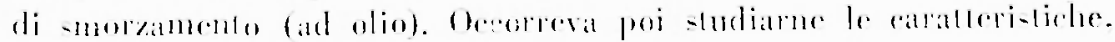

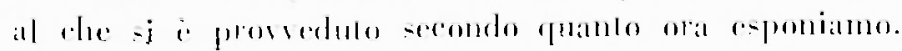




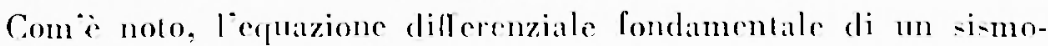
grafo si puo serivere, indieando con a lampieza della regi-trazions c ron $x$ lo yostamento del suolo (fuindi con $x "$ l'arelerarione):

$$
a^{\prime \prime}+2 x a^{\prime}+n^{\prime \prime}=-V_{n} x^{\prime \prime}
$$

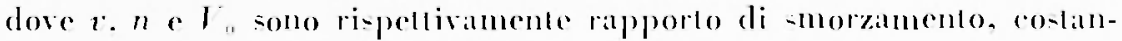

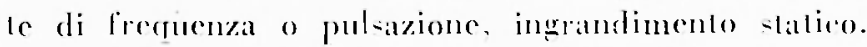

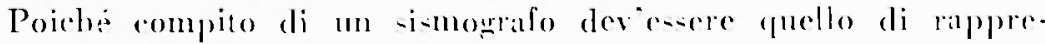
sontare in maniera conforme il movimento del suolo. lat pl] indirat che rio si verifieherehbe per un -ismogralo per rui lowe

$$
2 r a^{\prime}+n^{2} a=o
$$

perché allora ammellendo dre all istanle iniziale $1=0$ sia $x=0$ e $a=o$, e quindi nulle le costanti d"integrazione, si arrohb:

$$
a=-1_{0} x, \operatorname{cis} x=-\frac{a}{V_{0}} .
$$

Si olterrebhe rosi direllamente lo spostamento del suolo.

La condizione [2] non is pero praticancente realizzabile (perehta non si puo anmullare lo smorzamento e la frepuenza). per ani -i is

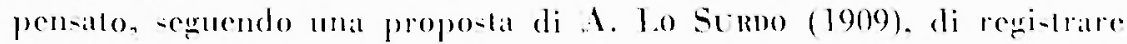
direllamente almeno lacelerazione. ponendo

$$
n=\text { molto grande }\left(\text { ios, } T_{n} \text { molto pireolos } \quad[3]\right.
$$

in modo di poter con-iderare traturabili i primi due termini debla [1] ri-petto al terzo, perehé allora la [1] -i ridure a

$$
n^{2} a=-V_{0} x^{\prime \prime}
$$

dal rui

$$
x^{\prime \prime}=-\frac{n^{2}}{l_{0}} \cdot a
$$

Nel caso in esame, is $T_{1}=0.37:$ quindi $n=16.7$ rel $n^{\circ}=280$. In prima approwimazione. i puó fuindi ritenere valida la ! 1]: lo - trumento è quindi un arcelerometro.

Oceorre ancora. per ulilizzare la 117 . il ralcolo di $1 \ldots$

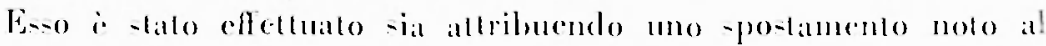


centıo di gravila della massal, ia mi-urando i rapporti d’ingrandimenlo dei vari sislemi di leve; $\dot{e}$ risullato $r_{4} \sim_{250}$

Risulta quindi, ron sufficiente approsimazione,

$$
x^{\prime \prime} \sim \ldots,
$$

rioè an l mu di ampieza registrala corri-ponde l'acrelerazione di 0.1 gral $=1 \mathrm{~mm} / \mathrm{rer}$.

Lo scorrimento, regolabile entro limili molto valiti, is stato portato al masimo, dala la piecoleza dei periodi da regiatrare: più precisamente si is aruto:

$$
1 \cdot 111-1.81: 60
$$

Pur enendo eonto die i di-poneval dellat sola componente ver-

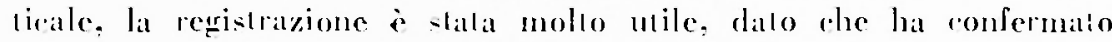
chiaramente numerosi impulei.

Lal fig. 2 riporla mo ingrandimento della registrazione numero 6.

Le co-lanli - trumentali crano le escuenti nell'aprile 1947:

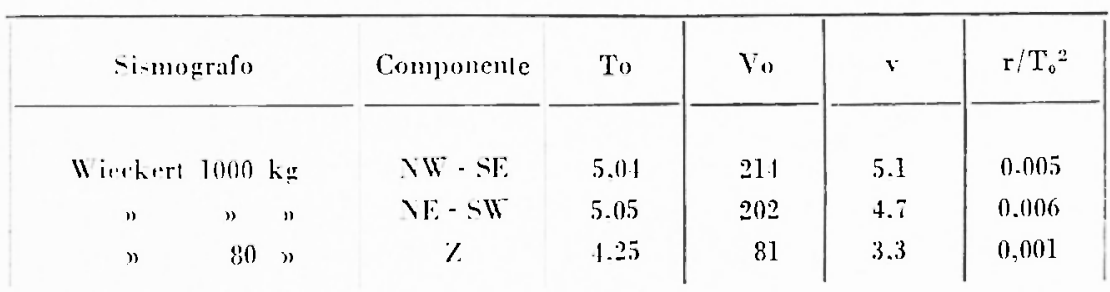

\begin{tabular}{|c|c|c|c|c|c|c|c|}
\hline$\| \mathrm{i}_{\mathrm{i}, \mathrm{k}}$ & 1000 & & $N W \cdot S E$ & 5.0 & 208 & 4.5 & 0.006 \\
\hline 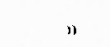 & $"$ & & $N E \rightarrow W$ & 5.0 & 200 & 4.4 & 0.006 \\
\hline " & & " & Z & 1.25 & 80 & 3.3 & 0.001 \\
\hline Illiani & & 》) & $E-\mathbb{W}$ & 12.5 & 1700 & $x$ & folo: $=-1 m]$ \\
\hline
\end{tabular}

Y.l marzo 1918 ene crano:

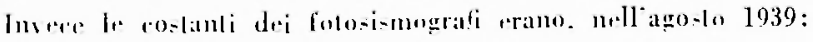

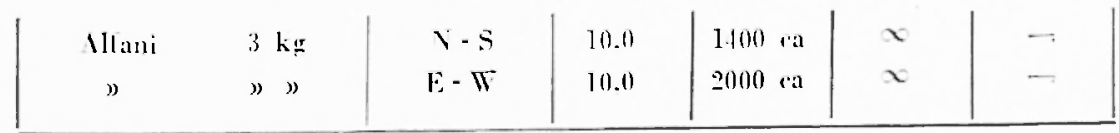




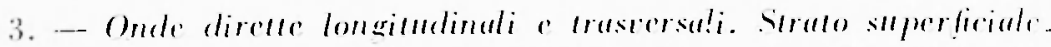
l.e differenze d, dei lempi di atrivo delle onde tra-versali e di quelle"

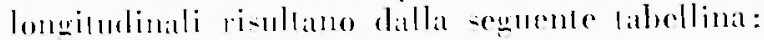

\begin{tabular}{|c|c|c|c|}
\hline Yant. & $\underset{(l: m)}{\operatorname{Wi-(anzal}} D_{i}$ & $\frac{d \mathrm{i}}{(\sec )}$ & $l_{\mathrm{i}} \quad D_{\mathrm{u}}$ \\
\hline 1 & 3.1111 & $11 . \overline{.}$ & 10.2 .131 \\
\hline 3 & 3.70 & - & - \\
\hline 1 & $3.8 \pi$ & 111.81 & 10.2010 \\
\hline 5 & 1.6 .5 & 1.11 & $0 . .21 .5$ \\
\hline 6 & 5.65 & 1.2 & $11.2: 211$ \\
\hline$\tau$ & 6. 18 & $1 . .8$ & 11.201 \\
\hline$i$ & 9.60 & 2.11 & $11.211 \%$ \\
\hline 1) & 16.23 & 3.25 & 01.21111 \\
\hline 111 & 16.20 & $\because \because$ & $0.1 \div 0$ \\
\hline
\end{tabular}

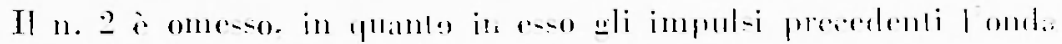

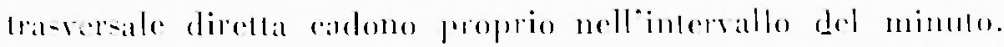

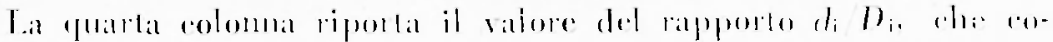

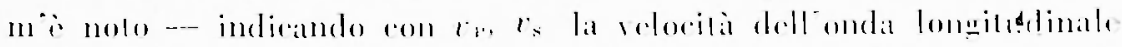

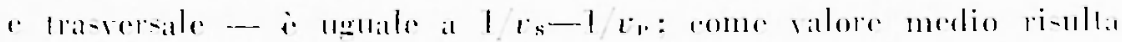

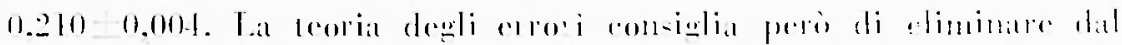

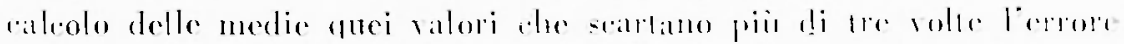

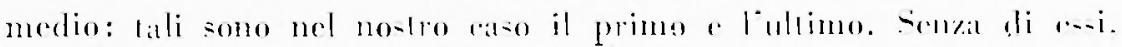
il valore medio diventa:

$$
\frac{d}{l)}=0.2085,0.0132=\frac{1}{r_{s}}-\frac{1}{r_{1}} .
$$

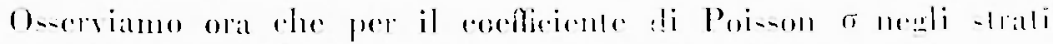

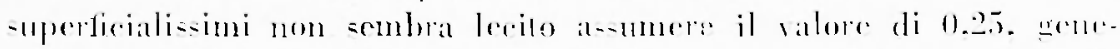

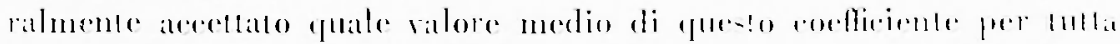

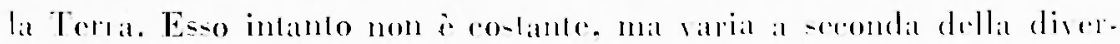

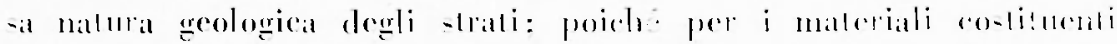
gli strali più intemi sono sali loovali valori anche molenolmente -

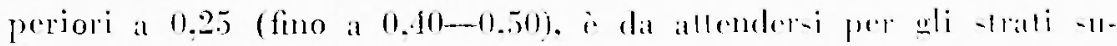

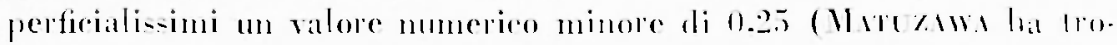
valo. per esempio. il valore 0,17).

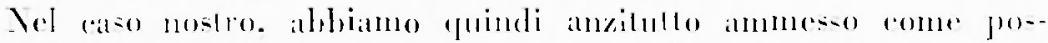

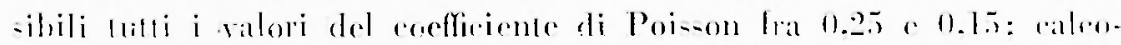

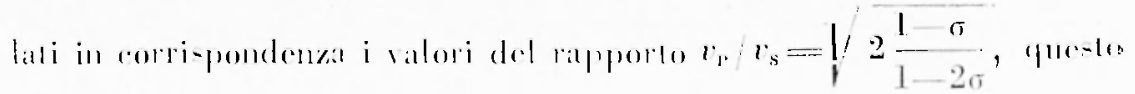



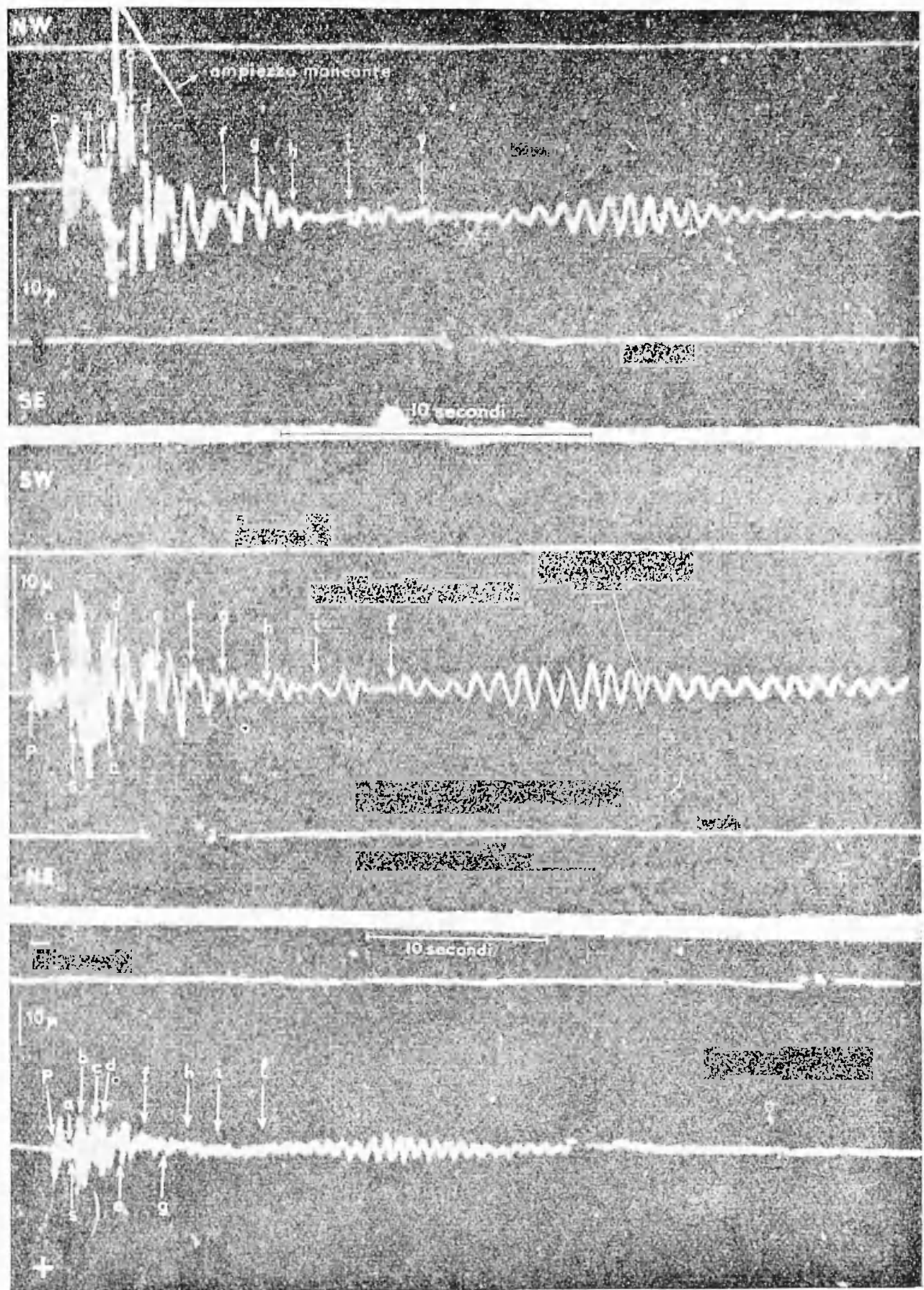

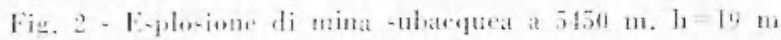


ci hat fornito. mitamente alla relaxione precedente, $i$ valori di re c es. I valori numerici sono riportali nella labella che senue:

\begin{tabular}{|c|c|c|c|c|c|}
\hline$\sigma$ & $i_{1}, r_{s}$ & $r_{p}$ & $r_{s}$ & $r_{b}$ oss. & $r_{h} \cos r$ \\
\hline 0.25 & 1.732 & $35 ! 2$ & 20128 & 1802 & 1739 \\
\hline .23 & .689 & 3306 & 1956 & 175. & 1695 \\
\hline .21 & .652 & 3129 & 1891 & $16 \% 5$ & 16.39 \\
\hline 19 & .616 & 2951 & 1838 & $1630^{\circ}$ & 1587 \\
\hline .17 & .580 & 2811 & 1795 & 1.587 & 1538 \\
\hline .15 & .559 & 2681 & $1 \div 21$ & 15.55 & 1508 \\
\hline
\end{tabular}

I valori delle velorilà sono (yui a nel seguilo) in 11 -ere.

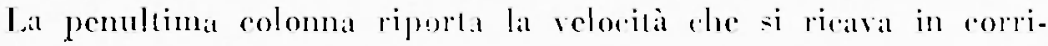
spondenzal per un partieolate lipo di onda registratal (indieatal ron

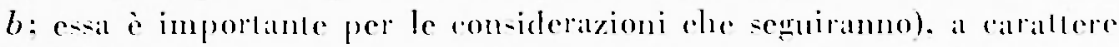
longiludinale e eon tragilto nella strato di mare fra pumto di -roppio

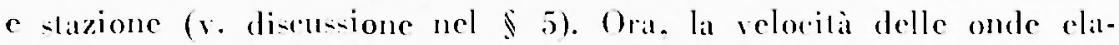

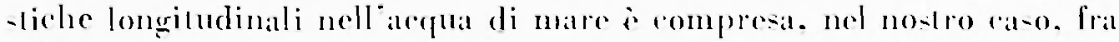
1480 e $1490 \mathrm{~m} / \mathrm{sec}$. Considerando che lultimo tratto del tratrilto periorso prina di arrivare alla stabone de di 250 m in lermaferma dore

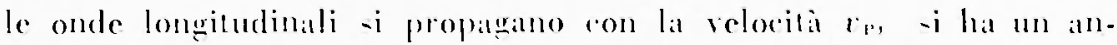
lieipo nel tempo di arrivo di 0.2 sece. Tenendo conto di questa correzione, -i hamno (per la di-lanza di $70 \mathrm{~km}$ ) i valori di is riportanti nell'ulima colomma della preedente tabella.

L'ullimo valore i praticamente coincidente con quello della re. locilà delle onde longiudinali nell acqua. Per que-lo motivo siamo indolli al ritenere che il valore piit probabile per il roeffiente di Poisson nello slrilo -uperfuciallisinno -ial

$$
\sigma=0,1.5
$$

c quindi in rorri-pondenzal

$$
\imath_{1}=2680 \mathrm{~m} / \mathrm{sec}, t_{\mathrm{s}}=1.20 \mathrm{~m} /-\mathrm{cc} .
$$

Dalo die, come vedremo piò atanti. la ma-sima profondita rategiunta dal raggrio simico dirello. dal punto di soppio allal Stazione e di poche decine di m. lo - trato altraversato è erelu-ivamente quello delle arenate e marne del fly-eh, an prevalenza delle arenarie fuella parle superiores. I ralori -opra olfenuli per la velocila delle onde lonegitudinali a law-rerali sono quindi in acordo eon i valori medi eneralmenle ammesi (REach-Zwersir. 194:3) per le arenarie fper

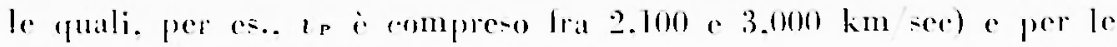
marne (fra 1.801$)$ c 2.400$) \mathrm{km}-\mathrm{-ec})$. 
Lat rono-en\%a dellia den-iti delle arenarie $e=2.65$, ensente di riratrate immedialimente -alla bate dei ri-nitali precedenti $i$ ralori detle altere co-tanti fi-iehe ed elatiehe delle trallo all raversato:

a) Vordulo di rigiditì:

$$
\mathrm{u}=0 . r_{\mathrm{s}}{ }^{*}=-.9,2.10^{10} \text { dine. } \cdot \mathrm{m}^{*}:
$$

1) Wodulo di Young:

$$
l:=11(2 \cdot 2 \cdot)=18.21 .10^{11} \text { dine } 1 \mathrm{~m}^{2}:
$$

c) Costemi di Lame:

$$
\begin{aligned}
& i=\frac{l:}{(1+\sigma)(1-2 \sigma)}=01.34 .1011^{\prime \prime} \text { dinc cm" } \\
& \iota=7.92 .161^{\prime \prime \prime} \text { ! }
\end{aligned}
$$

(1) Coeffirionte atincompresibilitit:

$$
K=\lambda+\frac{2}{3} ! 1=5.62 .10^{\prime \prime} \text { dine } \cdot \mathrm{m}^{2} \text {. }
$$

Qnesti valori -ono in huon aweordo con fuelli generalmenle anmeni per le arenarie.

1. -.- Here onde registrate. Loro dromocrone. Come ri-ulta dilla

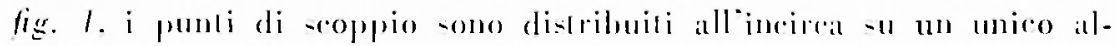
lineamento medio. indicato con una linea tratteggialia e corrispondenle ad un azmul di 325" rispello alla Slazione di Trie-le. Que-la cir-

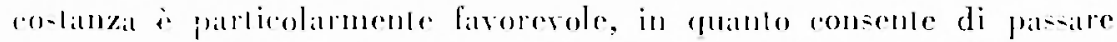
con magrator fiducia allia costuzione delle dromocrone per le divere

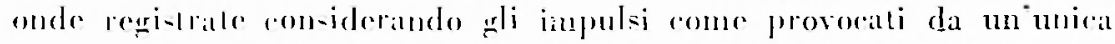

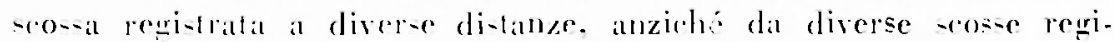

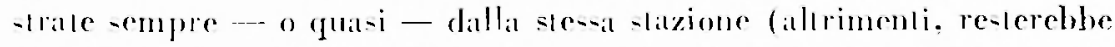

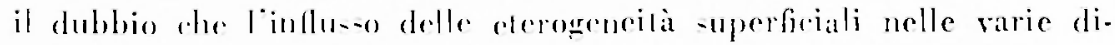
rezioni alleri il -innificalo fisien felle dromoerone -lesese).

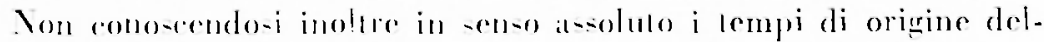

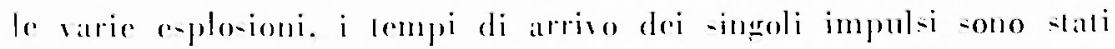
conlati a partire dal primo ( $P$ ). Le dromorerone risulanti sono riplprenentale nella fig. 3. a cono quindi relalive alla velocila di 2680

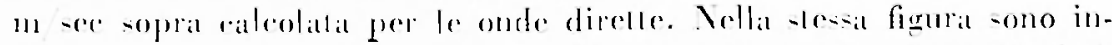
dirate ron $\rightarrow$ le onde lat-ser-ali direlle, thialdmente indiriduabili

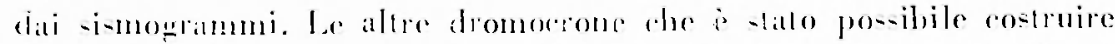
(per e-rrapolazione degli impulsi regi-labli) -ono indicate nella fig. 3

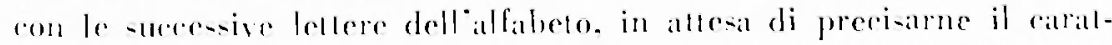
tere. Le dromorone a trillo piu malrato sono quelle che i riferi- 


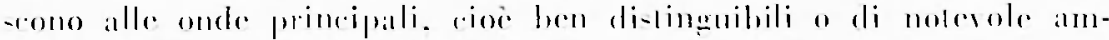

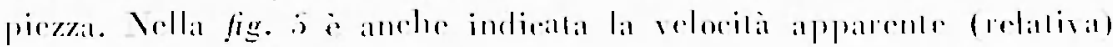

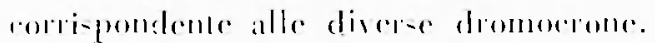

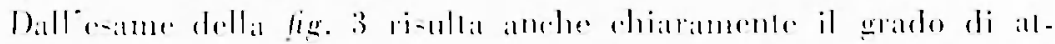

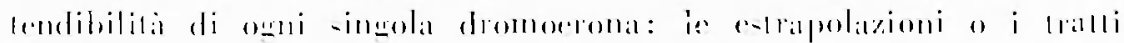

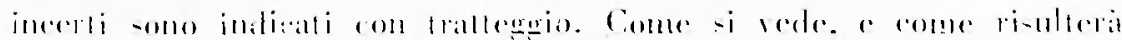

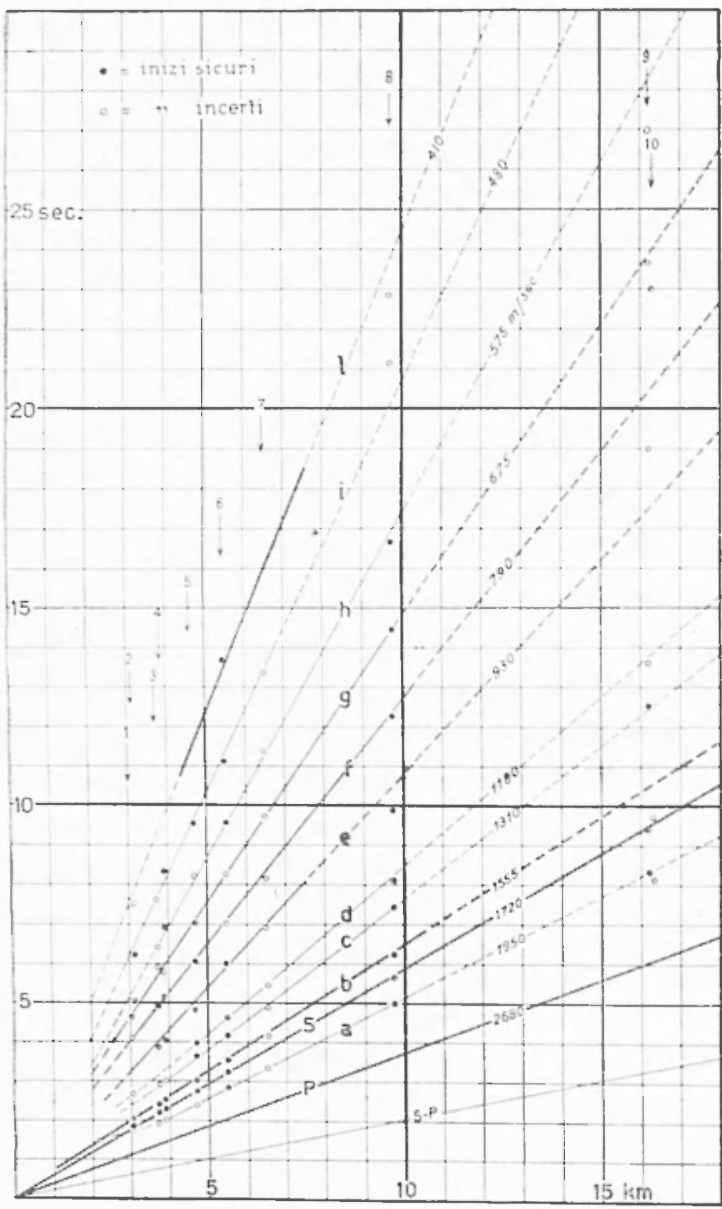

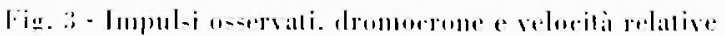
mewlio dalla di-an-io-

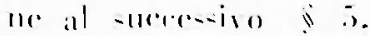
lallemditiliti i ollins: pere tulle le dromoremne principali, Iar ratr-il dell inlerelereal di alanuli llalli is lia EIr?ild:

Omla at. Ditriti-

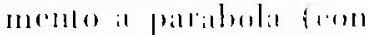
lal collatuila in allol nori prowi dellorieime

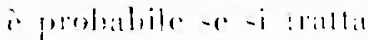

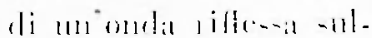
lat prima smperflireic di

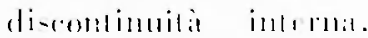

Omda b. II 1mallowein dopos i la hen in

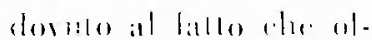
lare tale disansa nom -i allillo rermpli di rela-imi in mal:e: mal i. dis presedera ihe

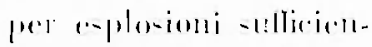
cincente inlenter lombla lomerublinale b-i pro-

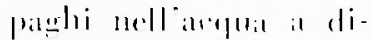
-latrze mangiori.

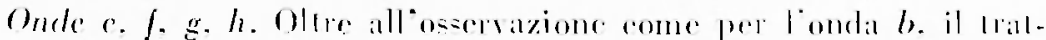

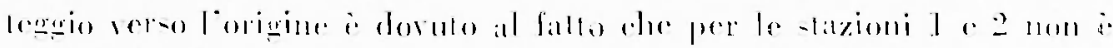

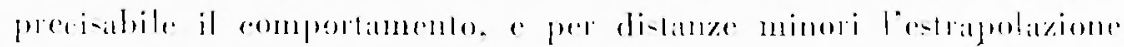

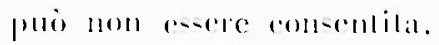




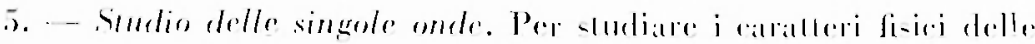

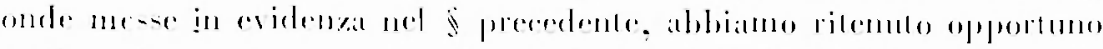
analizante una per ma netle diverse regi-lrazioni. E-poniamo ora

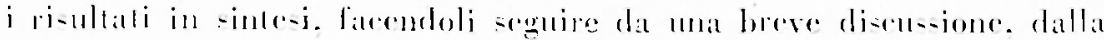

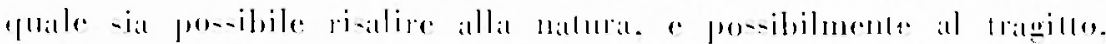
dell ondat -lenas.

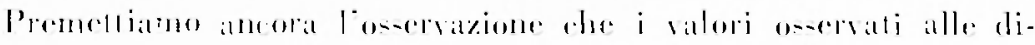
-lanze minori -omo da rilener-i ineerli per l'esignila dello soorrimenlo

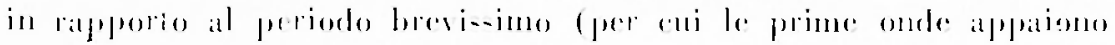

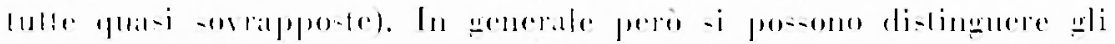
inizi e le ampleme delle la-i.

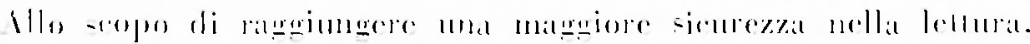

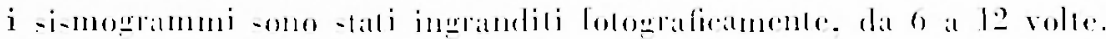

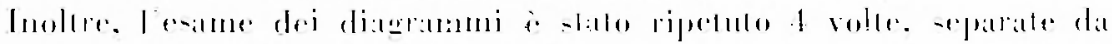

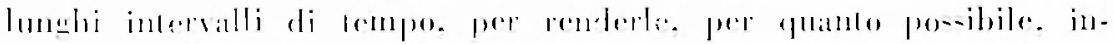
dipeist:rnli.

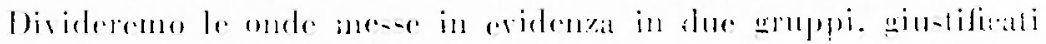

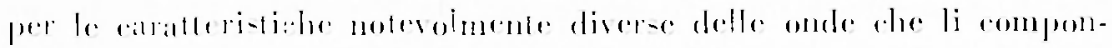

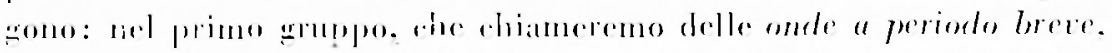

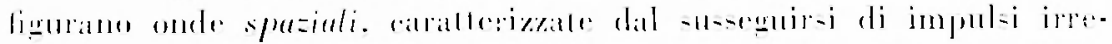

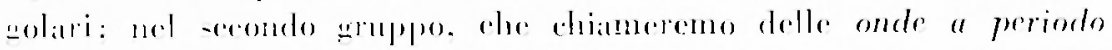

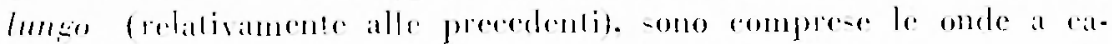

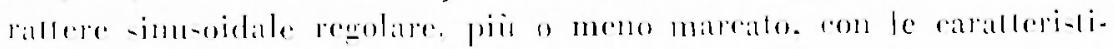
che delle onde superficiali.

\section{I. -- Onde a periodo hreve.}

Ondal'.

\begin{tabular}{|c|c|c|c|c|}
\hline \multirow{2}{*}{ Yum. } & \multirow{2}{*}{$\begin{array}{c}\mathrm{T} \\
\text { (sure) }\end{array}$} & \multicolumn{3}{|c|}{ \m|pie\%za :micron! } \\
\hline & & 111 & $\mathrm{Xl}$ & Z \\
\hline 1 & $|11.1|$ & 11 & ] & $?$ \\
\hline 2 & \multicolumn{4}{|c|}{ Vell"intersall, , lal mitnuts } \\
\hline 3 & 110.11 & $i$ & $\underline{2}$ & ? \\
\hline .1 & 111.11 & 6 & 2 & $?$ \\
\hline . & 0.1 & -1 & 2 & 0.8 \\
\hline 6 & 0.1 & (i & 2 & 1.2 \\
\hline ; & 0.1. & \multicolumn{2}{|c|}{ a } & 2 \\
\hline$\because$ & 0.18 & $0 . \vec{j}$ & Ir & $?$ \\
\hline 9 & 10.3 & 0.4 & 0.1 & $?$ \\
\hline 10 & ? & 一 & - & minatial \\
\hline
\end{tabular}


Con $T$ abbiamo indicalo il periodo dell'ondal.

Per i soli n. 5 e 6 istato posibile calcolare l'angolo di emertrenza apparente c.. dal quale poi si a ricavalo langrolo di energenza vero es mediante la nola formula:

$$
\cos e=\frac{v_{\mathrm{r}}}{v_{\mathrm{s}}} \sqrt{\frac{1}{2}\left(\operatorname{l-sen} v_{10}\right),}
$$

dove per $\frac{v_{r}}{v_{s}}$ ahbiano ammeso il valore 1.56 derivante dai valori sopra trovali per la velociti delle onde direlle longiludinale e tratsersale. F: risultalo:

$\begin{array}{clc}11 . & c_{01} & c \\ 5 & 10^{\circ} & 6^{\circ} \\ 6 & 11^{11} & 8^{\circ}\end{array}$

11 segno della componente verlieale is risultalo naturalmente sempre +- dalo ehe si lralla di esplosioni, che provocino -ul fondo an impulso verso il basso. e quindi alla slazione un impul-o reroo l'allo.

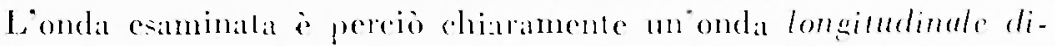
reta nello stralo superiore del flych. Pes arrivare inlatli dat punti di scoppo indicali. ques onda pereore tragilli molto pro-imi alla superfeic. per i quali cercheremo ora di caleolate almeno approw simalisamente la massima profondila ragramenta.

Ammellendo, come grenerilmente $i$ fa nella pro-pezione -i-mica. che la velocila ammenti linearmente eon la profondila h. a pareitr dal valore $b_{\text {a }}$ alla -uperficie. secondo la relazione

$$
\iota_{h_{1}}=v_{1}+h_{1} \cdot h,
$$

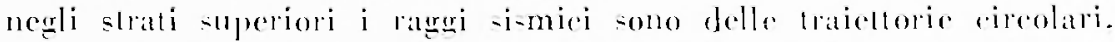

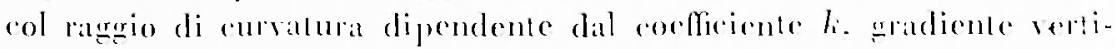

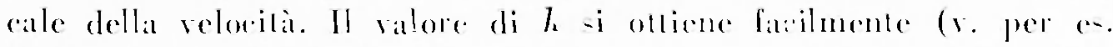

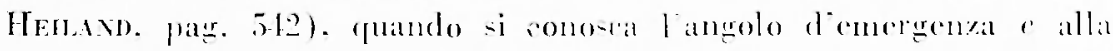
di-lanza $x$ della relazione:

$$
k=\frac{2 v_{n} \cdot t g \mathrm{e}}{x} .
$$

Applicando que-ta formula a due cati 5 o 6 -opra nominati. si olliene per entrimbi: 


$$
l=0.121 \text {. }
$$

Conosedulo $h$. La profondià di penetrazione $h_{p}$ del ragerio si riralsa immerlialamentr dialla formulas:

$$
h_{\mathrm{p}}=\frac{v_{\mathrm{o}}}{i} \frac{1-\cos e}{\cos e} \text {. }
$$

Olleniand ro-i noi due rat-i com-irlerali:

$$
\begin{aligned}
& \text { per } x=46.50 \mathrm{~m}: h_{\mathrm{p}}=112 \mathrm{~m} \mathrm{ra}, \quad r_{p}=2095 \mathrm{~m} \text { see: } \\
& \text { per } x=5.50 \mathrm{~m}: h_{p}=1.56 \mathrm{~m} \text { a. } \quad v_{p}=2700 \mathrm{~m} / \mathrm{sec} \text {. }
\end{aligned}
$$

T'eniamo ancoral a preeisare che que-li valori derono inlender-i -ollanlo come indealivi dell'ordine di grandeza delle varie quanlili

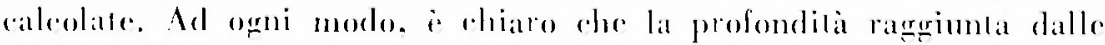

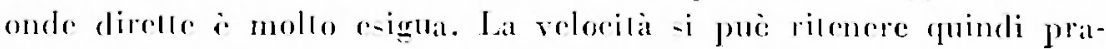

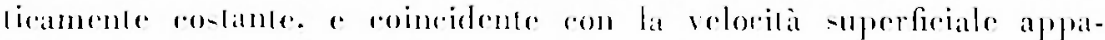

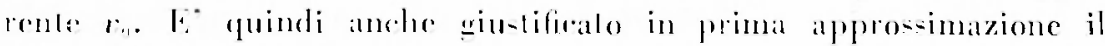

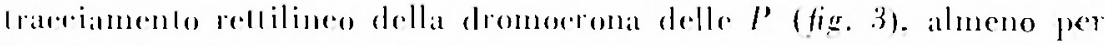

\begin{tabular}{|c|c|c|c|c|c|}
\hline \multirow{2}{*}{$\backslash 1111}$. & \multirow{2}{*}{$\begin{array}{c}\text { jo } \\
(\sec )\end{array}$} & \multicolumn{3}{|c|}{ Ampierza (micron) } & \multirow{2}{*}{$a P$} \\
\hline & & $N W$ & $\checkmark E$ & $Z$ & \\
\hline & \multirow{2}{*}{\multicolumn{5}{|c|}{ 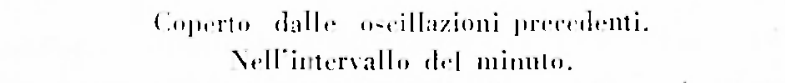 }} \\
\hline 2 & & & & & \\
\hline 3 & 10.21 & 111 & 111 & (9) & 1.1 \\
\hline 4 & $(0.21$ & $\| 1$ & 161 & 17 & 2.7 \\
\hline 5 & $(11.2)$ & $1 \cdot n_{1}$ & $\mid 1.31$ & 121 & 0.8 \\
\hline 6 & 111.21 & i & 2 & $1 \mathrm{r}$ & 11.6 \\
\hline$\imath$ & 0.1 & \multicolumn{2}{|c|}{111 a 11 व } & tr & - \\
\hline 3 & $?$ & 1 & 11 & tr & 一 \\
\hline 9 & 11.1 & $11 . \overline{-5}$ & $\operatorname{tr}$ & $1 \mathrm{r}$ & - \\
\hline 10 & $\because$ & - & - & m & - \\
\hline
\end{tabular}
le piecole distanze eon-ideral:"

\section{Cinda $a$.}

Gli clemenli onemali -ono ria-wuli nolla labella seguenle:

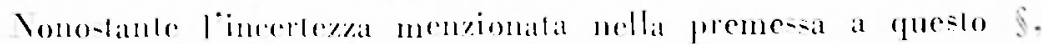

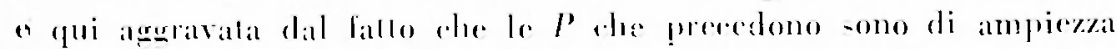

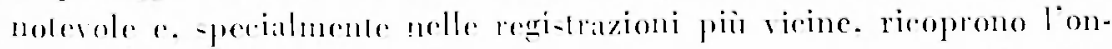

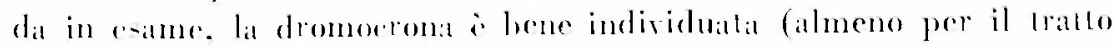

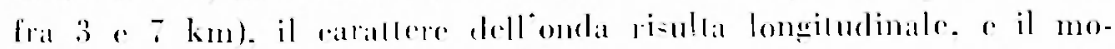

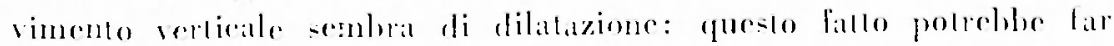

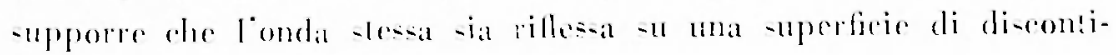




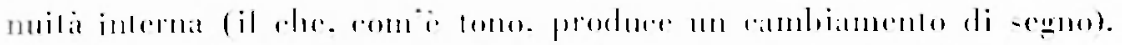

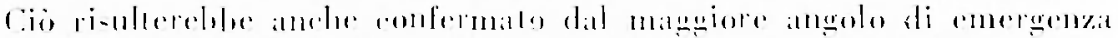

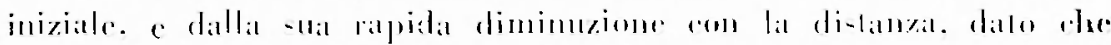

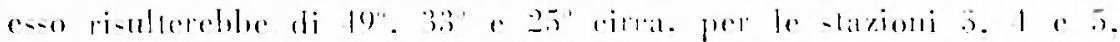
ri-petliviminalle.

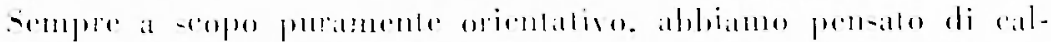

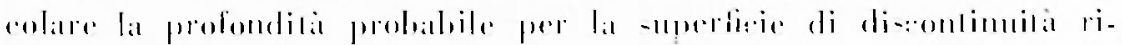

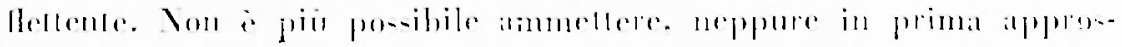

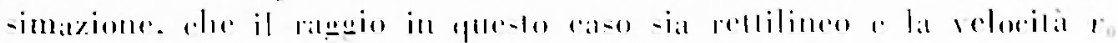

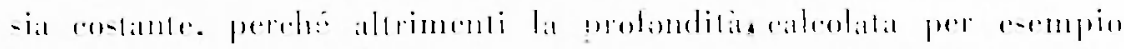

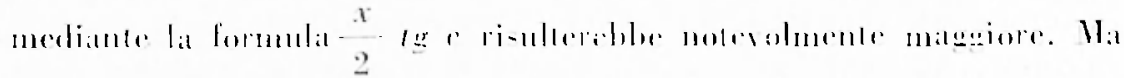

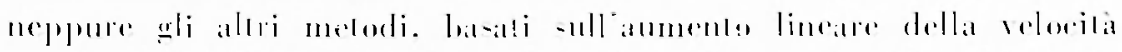

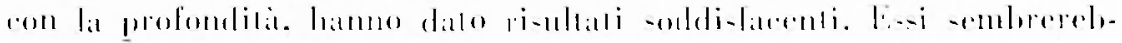

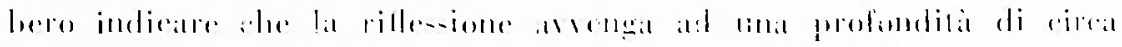

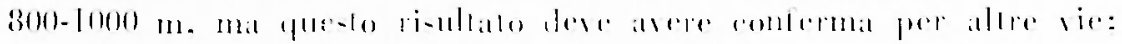

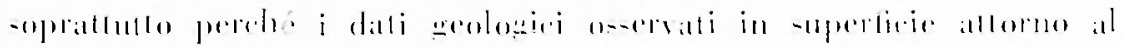

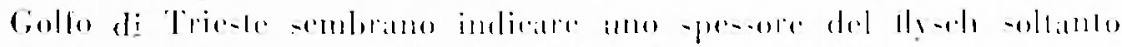

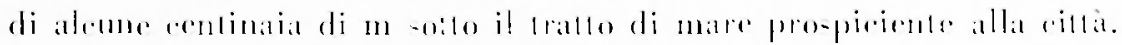

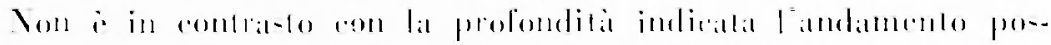

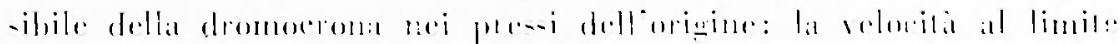

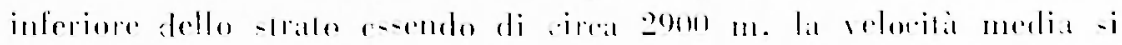

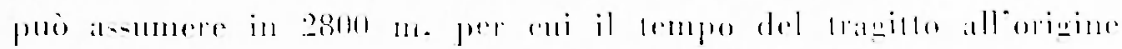
-ilrelilu di 1.4 - $=$ er.

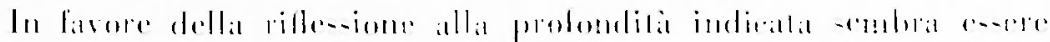

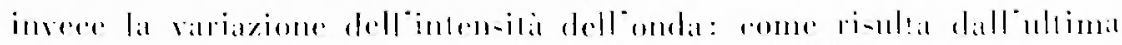

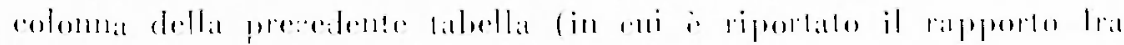

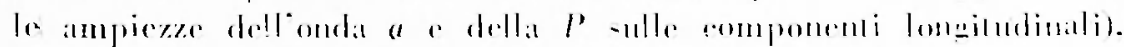

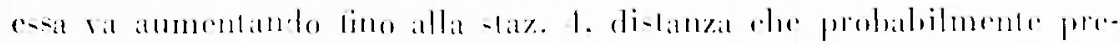

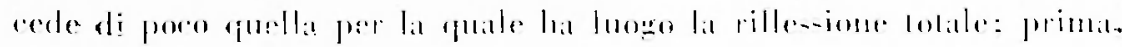

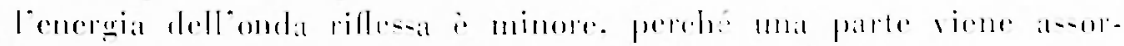

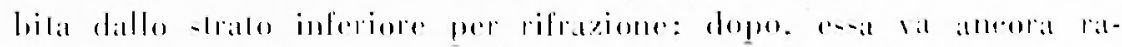

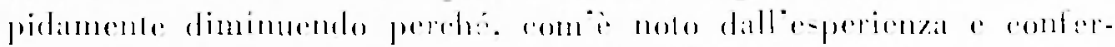

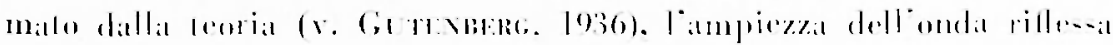

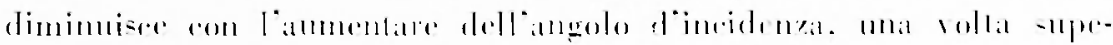
ralo limeolo limite.

\section{Ondit?.}

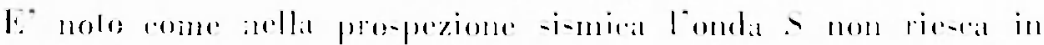

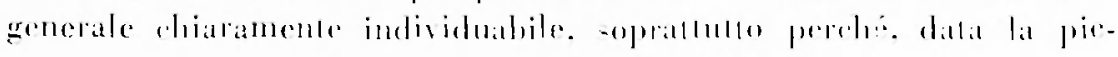


colezza della distanza, lintruallo di lempo dalla $P$ e troppo breve.

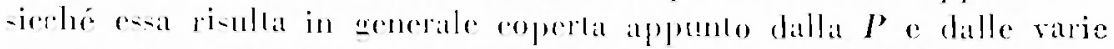
riflesioni e rifrazioni.

Velie regi-trazioni pui studiate la fortumala coincidenza di avere

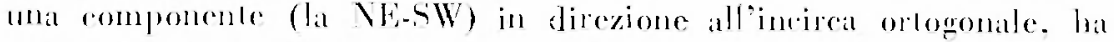

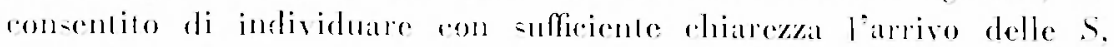
atppunlo st rewe-tal romponente.

Riportiamo, eome per le onde precedenti, il periodo e le ampiczze oncriali:

\begin{tabular}{|c|c|c|c|c|}
\hline \multirow{2}{*}{ Vun. } & \multirow{2}{*}{$\frac{\mathrm{T}}{\left(\sec ^{2}\right)}$} & \multicolumn{3}{|c|}{ Ampieza (micron) } \\
\hline & & $\lambda W$ & $\mathrm{NE}$ & $\mathrm{Z}$ \\
\hline 1 & ? & ? & 26 & - \\
\hline 2 & \multicolumn{4}{|c|}{ Nell’intervallo del minnto } \\
\hline 3 & 0.1 & (अ) & 13 & $\mathrm{tr}$ \\
\hline 4 & 0.2 & ! & 5 & - \\
\hline 5 & 0.2 & - & 1 & - \\
\hline 6 & 0.2 & ? & 1.5 & 2 \\
\hline 7 & - & & & - \\
\hline 8 & 0.2 & 3 & 2 & 1 \\
\hline 9 & (10.15 & 10.1 & 0.1 & 0.1 \\
\hline 10 & $?$ & $?$ & 0.5 & 111 \\
\hline
\end{tabular}

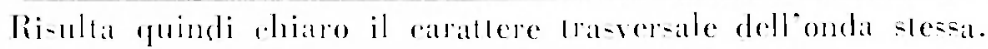

Oiralab.

Per lo sazioni lino al $n$. 6, abò per distanze almeno fino a

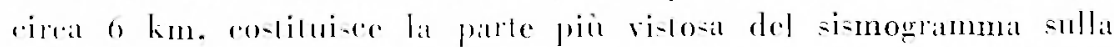
romponente longitudinale c - - guella verticate. Ciò risulta chiaramente dalla seguente labella rele riporta i valori o-ecrati.

\begin{tabular}{|c|c|c|c|c|c|}
\hline \multirow{2}{*}{ Xum. } & \multirow{2}{*}{$\begin{array}{c}1 \\
(s+c)\end{array}$} & \multicolumn{3}{|c|}{ Lmpirzar (micron) } & \multirow{2}{*}{$b \cdot \mathrm{I}^{\prime}$} \\
\hline & & $\mathrm{NW}$ & $\mathrm{VE}$ & $Z$ & \\
\hline 1 & 11.1 & 5.3 & 24 & 1241 & 5 \\
\hline ? & $?$ & 5 & 22 & 12 & - \\
\hline 3 & 0.1 & 11 & 17 & 8 & 6 \\
\hline 4 & 0.1 & (1.10) & 20 & 9 & $i i$ \\
\hline 5 & 0.1 & 30 & 10 & 3 & $i$ \\
\hline 6 & 10.1 & 111 & 9 & 161 & 7 \\
\hline$\tau$ & 11.15 & & & 1 & - \\
\hline 8 & 0.15 & 2 & 1.5 & $\operatorname{tr}$ & 2 \\
\hline 9 & - & $?$ & $?$ & $?$ & - \\
\hline 10 & - & - & - & $\pi$ & 一 \\
\hline
\end{tabular}


Ri-ulta intanto exidente il calratlere lomgitudinale di qua-t'ondat.

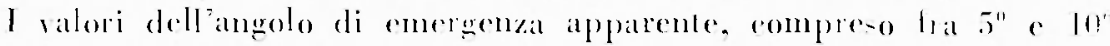
(il che rorrisponde ad an angolo rero molto piccolog. e la noterolissima ampievar alle pireole distanze, stammo a dimostrare ehe si tralta di unonda longitudinale propagantesi direntamente mell arqua. Ciis

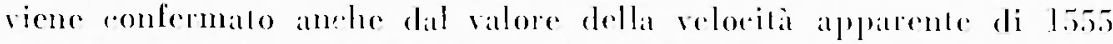
m. aee cui rortisponde. come abbiamo visto nella di-ew-ione al $\$ 3$. una velocita effelliva di rimal $1500 \mathrm{~m}$ m/-ore.

In renclusione, si tratla di monda di romprentone e diatarione,

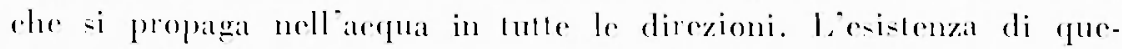
st onda é confermata dalle osecrazioni dirette -ul mane nei pressi delle esplosioni: pochi islanti doper abbarata la leva per il brillatmento eletrico a diatanza. si arvertiva il "colpo sul fondon delJimbareazione aleompagnato, per un tempo molto breve, da rapide occillazioni, col mate perfellamente immobile: nel riso del rimorchiatore, di co-truzione motalliea, ese -i traducerano in vibmpioni di tutte le parli con notevole rumore. Suene-ivamente appenat. si

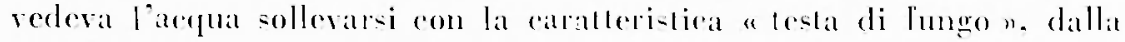
quale infue sprizzalval nel entro il calralleri-lico eonello rhe. -imile ad un greycer, rag̣imgera altezze anche di $70-80 \mathrm{~m}$.

Investendo la rosta, que-le onde longitudinali moltiplicano il low affello. e cio spiega la noterole ampiezal delle registrazioni nei ra-i di esplosioni in mare. Inversamente, anche nel rato di eplosioni in terra prossime alla eosta \& po-sibile rhe onde di que-lo lipo -i propatghimo all'adqua, ma naturalmente leintensiti sari molto minore. secondo quanto appunto e stato rontalito nelle rexistraxioni dei $n$. 9 e 10 per le quali l'ampiezar di questonda is ridoltissima o addirilluta mancal del tulto. rome nell ultimo ratoo.

Liultima colonna della labella precedente indiea $i$ valori del rapporto $b / P$ : da e-ei risulta rhe pratieamente lo smorzamento dellonda e traseurabile almeno fino a cirea $5 \mathrm{~km}$ di disanza dall"origine (inrece la diminuzione di ampiozza della $P$ io già semibile). I dali a disposizione non roncontomo ulteriori deduzioni per di-lanze margiori.

Queste onde posono subire riflessioni. ia sullat superlieie libera del male, sia sul fondo: ma di puese parleremo fral poeco.

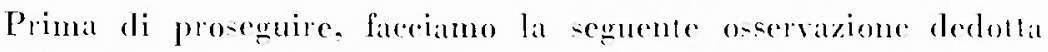
dalla fig. 3: nessuna delle dromoerone ohe seguono sembra arere un"inclinazione minore della precedente, rome doveblue exere nel 
raso ele qualema di ese si riferiece a onde rifratte in uno strato inferiore, con velocita quindi comsibilmente magerore di fuella rui trovata per le $P$ nel flyorlu. Le onde mesce in evidenza sono quindi lubte onde riflesse. Il ahe può essere in areordo con i dati che la Grologia ammelte per la statifieazione superficiale nella zona in éame: lo strato del fysch doveblbe avere, come gria detto, uno spe-ore di qualche rentinaio di $m$, ed esere seguilo dai calcari (ne)lordine: del Cretaceo sup., inf., del Giurese). Per rui, con i dati di-poniliti, rle praticamente sono sulfieientemente densi solo fino a riral $6 \mathrm{~km}$ (le regitrazioni 8. 9 a 10 devono intendersi più che altro valide per lestrapolazione dei dati messi in evidenzil con le regisrazioni piir vicine), e con il piecolo potere risolutivo dei sismogrammi, non i posibile mettere in evidenza eventuali onde rifratle newli -trati inferiori, relatisamente troppo profondi.

\section{Onder}

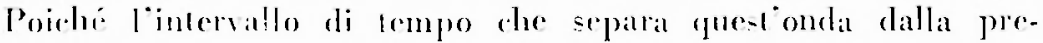
redente a molıo breve, exa is di dilicile individuazione, specie alle di-tanze minori, ri-ultando coperta dalle (ampic) oscillazioni dell'ondat $b$. Somo pereio molto incerti i dali di oservazione, che oral riporliamo:

\begin{tabular}{|c|c|c|c|c|c|}
\hline \multirow{2}{*}{ Num. } & \multirow{2}{*}{$\begin{array}{c}\mathrm{T} \\
(\mathrm{s}, \mathrm{N})\end{array}$} & \multicolumn{3}{|c|}{ Ampiezza (micron) } & \multirow{2}{*}{$\cdot / \mathrm{P}$} \\
\hline & & $\mathrm{NW}$ & $\mathrm{NF}$ & $\mathrm{Z}$ & \\
\hline 1 & \multicolumn{4}{|c|}{ Copertal dallit precedente } & - \\
\hline 2 & & " & $"$ & & -- \\
\hline 3 & $(0.2)$ & ? & $\pi$ & (11) & - \\
\hline 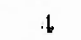 & 10.21 & 1201 & $?$ & $111)$ & 131 \\
\hline 5 & $(0.2)$ & $?$ & 2 & $?$ & - \\
\hline 6 & 0.1 & 191 & 5 & $?$ & 2 \\
\hline$\tau$ & ? & & & 1 & 一 \\
\hline 8 & $?$ & 1 & $?$ & 1 & 2 \\
\hline 9 & 0.4 & 1.5 & 1 & tr & 3 \\
\hline 10 & $?$ & $?$ & ir & m & - \\
\hline
\end{tabular}

Il s-mogramma del n. 9 indica ehiaramente che si tralta di unonda longrindinale. Esoa ha notevole ampieza anche per le esplosioni in teras, percio il tragitto non doverbbe essere nello strato d'acquat. fat romponente verlicale combra esere piuttosto sensibile. per eni 


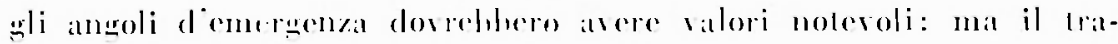
gitlo dellonda sesea non ímeglio precisabile.

\section{Omla d.}

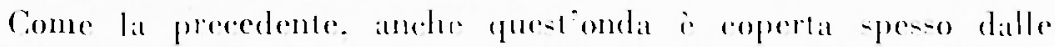
oscillazioni precedenti. I ditli di oscrvatione sono:

\begin{tabular}{|c|c|c|c|c|c|}
\hline \multirow{2}{*}{ Yum. } & \multirow{2}{*}{$\begin{array}{c}1 \\
(\sec )\end{array}$} & \multicolumn{3}{|c|}{ Amuni+rnai (micrmn) } & \multirow{2}{*}{ d 1} \\
\hline & & 1.11 & $V E$ & $\%$ & \\
\hline 1 & $(11.2)$ & $(.11)$ & 111 & 21 & $(1)$ \\
\hline$\because$ & \multicolumn{4}{|c|}{ Von disintruiluil, } & - \\
\hline 3 & \multicolumn{4}{|c|}{$n$} & - \\
\hline 1 & \multicolumn{4}{|c|}{$n$} & - \\
\hline$\overline{5}$ & 11.3 & 3 & - & 1 & 1 \\
\hline 6 & 11.1 & 5 & 2 & (, & 1 \\
\hline$\tau$ & $(11.2)$ & & & 12 & \\
\hline 8 & 11.1 & 3 & $\therefore$ & 3 & \\
\hline 9 & 111.51 & 1 & $0 . .5$ & $\pi$ & \\
\hline 10 & $?$ & $?$ & $?$ & 11 & -- \\
\hline
\end{tabular}

Londa presenta gli slesi caralleri della precedente. ron rom-

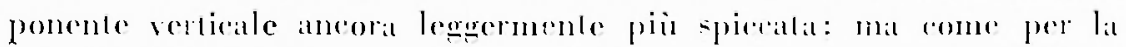
precedente il tragitlo non is preciabibile.

\section{1. - Onde a periodo lmngo.}

\section{Onduc.}

Tneomincia la serie alelle onde a ratallere regolate, -intu-oidale smorzato, con tulli grli aspetli delle onde -uperlediali del lipo co-iddetlo di Rayleigh per i terremoli lontani: risulta svihppata partiolamente sulle componenti longitudinale e verticale. in quatele re gistrazione sperialmente l'inizio ir matelierato da o-rillazioni a piu breve periodo, dei tipi precedenti. Lanizio is in particolar modo rhiaro nelle registrazioni dei n. 3 e 1 . Per le registranioni provenienli da esplosioni in terra perde quasi del tullo la-pello ali onda perfuciale, e si ridure ad aleme oscillaxioni di pircolat ampiezate piutlosto irrequari e di hreve periodo: rome se veniseso al mameare le onde a più lungo periodo che abbiamo qui con-ideralo, e restasero le onde a periodo breve dhe ad rese erino orrappose. 
Gli elementi osservati sono i segurnti:

\begin{tabular}{|c|c|c|c|c|}
\hline \multirow{2}{*}{ Vum. } & \multirow{2}{*}{$\begin{array}{c}\mathrm{T} \\
(\sec )\end{array}$} & \multicolumn{3}{|c|}{ Ampirazat (micron) } \\
\hline & & III & XF; & $Z$ \\
\hline 1 & \multicolumn{4}{|c|}{ Coprerla dalle precedenti } \\
\hline 2 & & 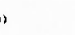 & $"$ & \\
\hline 3 & 0.6 & $i$ & 6 & ; \\
\hline 1 & 11.6 & (3) & $i$ & $\ddot{*}$ \\
\hline 3 & 11.6 & 5 & 3 & 2 \\
\hline 6 & $(11.7)$ & ! & 3 & 5 \\
\hline$i$ & (1).11) & \multicolumn{2}{|c|}{$11: a 11<a$} & 2 \\
\hline$\because$ & 0.5 & 3 & - & $\mathrm{tr}$ \\
\hline 1) & 111.31 & $11 . \overline{5}$ & 01.5 & Ir \\
\hline 10 & ? & $?$ & Ir & "I! \\
\hline
\end{tabular}

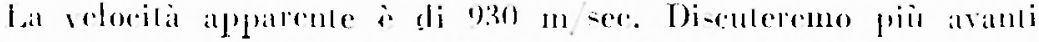
la po-sibile raura fi-ira ed il trigrillo di fuesta, e delle altre onde di puce-to !ruppos.

\section{Onda f.}

laleono le -tere oservazioni rome per l'omda precedenle: in piin $\therefore$ il latlo she in molli :ati is roperla anche dalla pre-

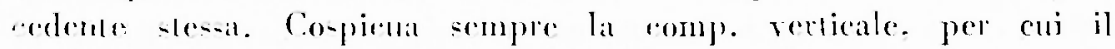

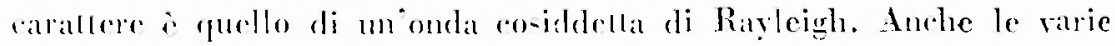
componenti romineiano ad carere fasale negli inizi, rome appunto -i onerra in enenerale nelle onde di Rayleigh. La velocita apparonle $\therefore$ di $790 \mathrm{~m}-\mathrm{ar}:$ eli clementi o-servali sono:

\begin{tabular}{|c|c|c|c|c|}
\hline \multirow{2}{*}{ Vum. } & \multirow{2}{*}{$\begin{array}{c}\mathrm{T} \\
\text { seces }\end{array}$} & \multicolumn{3}{|c|}{ A npiazzal (mecron) } \\
\hline & & 111 & $M E$ & $\%$ \\
\hline l & 11.7 & 3 & 9 & 2 \\
\hline$\ddot{2}$ & \multicolumn{4}{|c|}{ Coprerta da onde bresi } \\
\hline 3 & 11.8 & 5 & $?$ & $?$ \\
\hline 4 & 0.8 & 6 & - & ; \\
\hline $\bar{j}$ & $(11.5)$ & 3 & 3 & 5 \\
\hline 6 & 11.7 & 2 & 2 & ir \\
\hline i & (11.) $1:$ & & & $\stackrel{2}{2}$ \\
\hline : & 111.31 & 1 & 1 & $\mathrm{l}$ \\
\hline 9 & 0.6 & 1 & 11.5 & tr \\
\hline 10 & $?$ & $?$ & ir & m \\
\hline
\end{tabular}




\section{Orda $s$.}

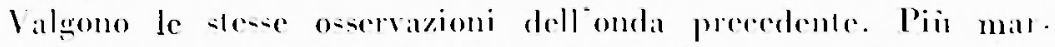
aata ancora la comp. verticale e l'ampieza della comp. longitudima.

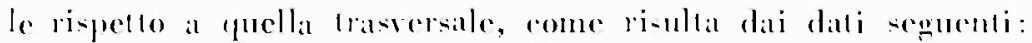

\begin{tabular}{|c|c|c|c|c|}
\hline \multirow{2}{*}{ Yum. } & \multirow{2}{*}{$\begin{array}{c}\mathrm{T} \\
\text { (sece ) }\end{array}$} & \multicolumn{3}{|c|}{ Ampierzal (micron) } \\
\hline & & $N W$ & $X F$ & $\mathrm{Z}$ \\
\hline 1 & 0.8 & 10 & 7 & 14 \\
\hline 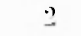 & 0,8 & 6 & 3 & 5 \\
\hline 3 & 10,81 & \multicolumn{3}{|c|}{ 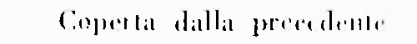 } \\
\hline 1 & 11.6 & \multicolumn{3}{|c|}{$n$} \\
\hline 5 & 0.5 & 3 & 1 & 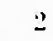 \\
\hline 6 & 0.6 & \multicolumn{3}{|c|}{ Coplecta da onde lomi } \\
\hline 7 & 0.1 & \multicolumn{3}{|c|}{$M$ a $n$ n } \\
\hline 8 & 0.6 & 0.5 & 11. & - \\
\hline y & 0.1 & $\mathrm{tr}$ & ir & - \\
\hline 10 & \multicolumn{3}{|c|}{ Coperta dalla preacedinte } & 川 \\
\hline
\end{tabular}

Vellanente dislingubile in quasi tutce le registrazioni il raralleri-

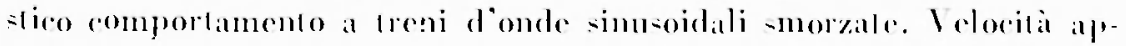
parente: $675 \mathrm{misec}$.

$$
\text { Orila h. }
$$

Caralleri e osscrabioni analoghi alle precedenti: ampjezza minore. Pobehé in molı casi è eoperta dalla precelente. non riportiame

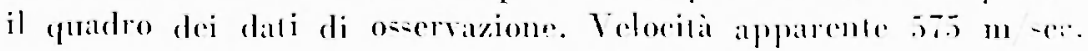

\section{Orala $i$.}

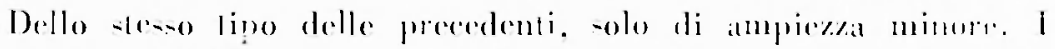
diti ri-ultamo dalla rewuente lalderlat:

\begin{tabular}{|c|c|c|c|c|}
\hline \multirow{2}{*}{ Nim. } & \multirow{2}{*}{$\begin{array}{c}\mathrm{T} \\
(\sec )\end{array}$} & \multicolumn{3}{|c|}{ Ampropral (micron) } \\
\hline & & NII & $X E$ & 7 \\
\hline 1 & \multicolumn{3}{|c|}{ 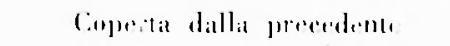 } & \\
\hline 2 & 0.8 & $?$ & $?$ & $?$ \\
\hline 3 & 0.8 & 3 & 2 & $?$ \\
\hline 4 & 0.8 & 2 & Ir & Ir \\
\hline 5 & 0.8 & 1.5 & 2 & - \\
\hline 6 & 0.7 & $0 . i$ & 1.5 & Ir \\
\hline 7 & 10.21 & \multicolumn{2}{|c|}{$M: 11110$} & 1 \\
\hline 8 & 11.5 & Ir & ir & Ir \\
\hline 9 & $?$ & $\mathrm{tr}$ & - & - \\
\hline 10 & $?$ & $?$ & $?$ & m \\
\hline
\end{tabular}




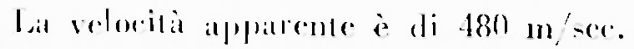

\section{Onder 1 .}

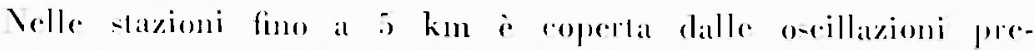

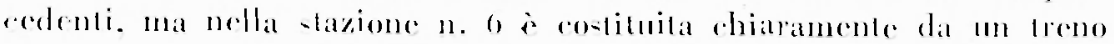

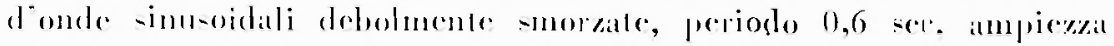

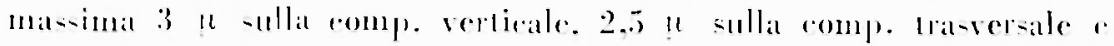

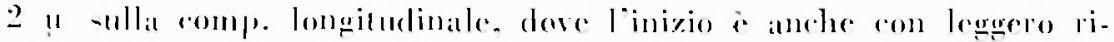

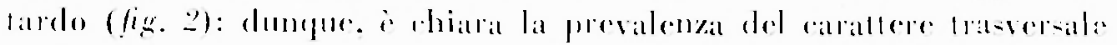
dellomelar trearat.

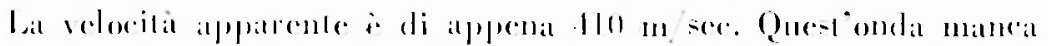
eompletamente per le epplo-ioni in tertale

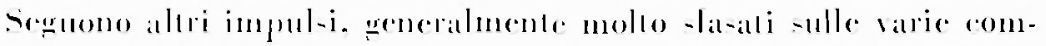
ponenti. noterolmente di-baneiali dalle onde fin yui ron-iderale, ma -remar un oreline apparente di di-lribuzione: poiche inolle ess sono

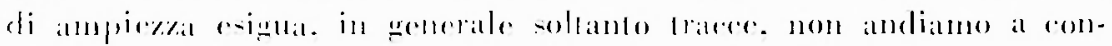
-iderarli.

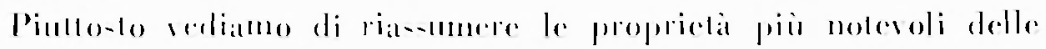

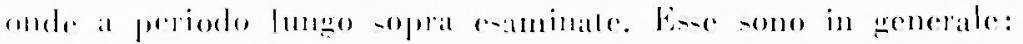

a) asperto pilllosto regolanc. " tremi donde sinusoidali smor-

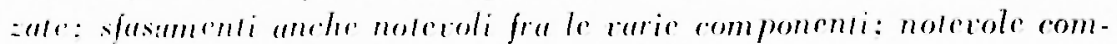

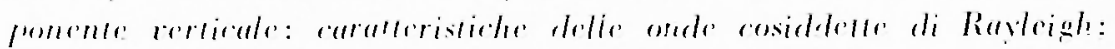

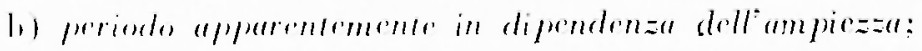

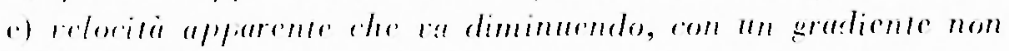
- ostanle ma dereresernte. in maniora quasi uniforme.

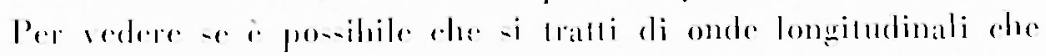

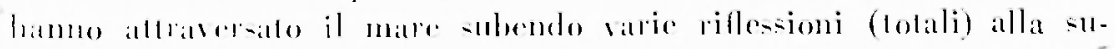

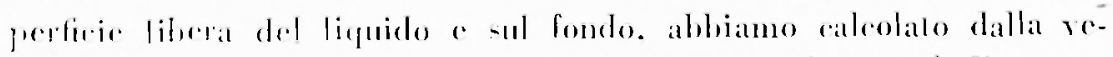

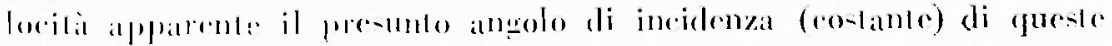
varie onde. ammellendo pere la velorita delle onde longeitudinali net-

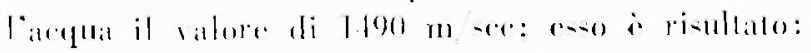

$$
\begin{aligned}
& \text { onda } 1: 39^{*} \quad \text { ondal h }: 233^{\prime \prime} \\
& \text { " } 1: 32^{\prime \prime} \text { " } \mathrm{i}: 19^{\prime \prime} \\
& \text { " } \because: 7^{-1} \quad " 1: 16^{\prime \prime}
\end{aligned}
$$

Poirhe l'angolo limile fra acequa e smolo per le onde sismiehe lon-

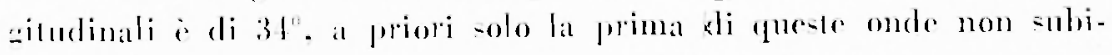




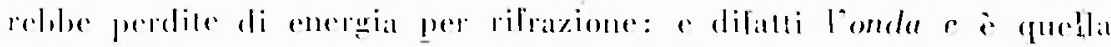
che ilat ri-pello alle allare ampiezze magegiori.

Ma lampiezza delle alue onder in paltieolare delle ullime, non

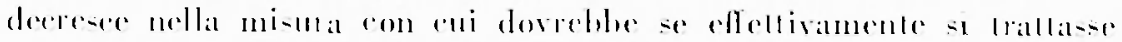

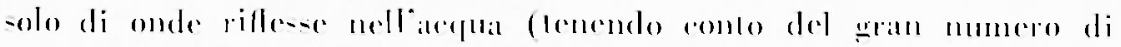

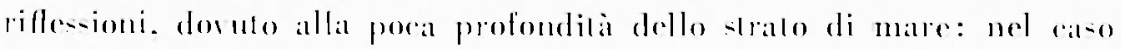

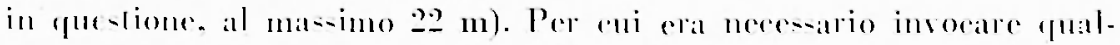

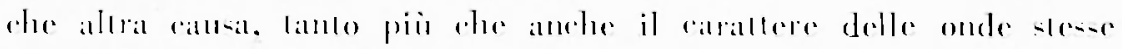
non e longiludinale.

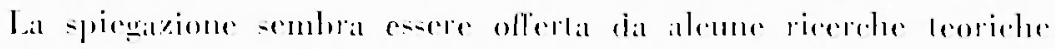

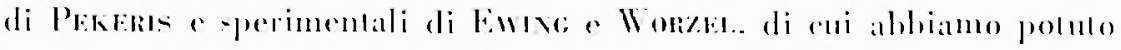

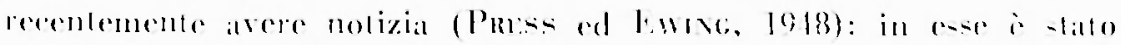

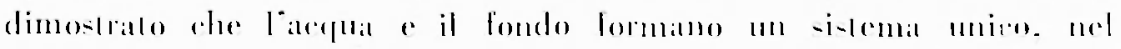

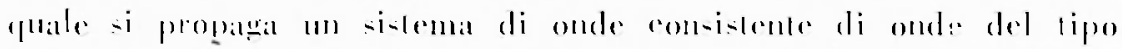

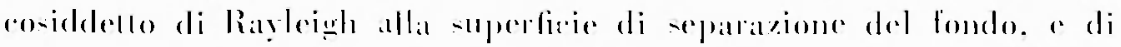

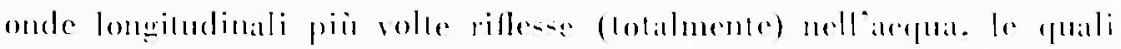

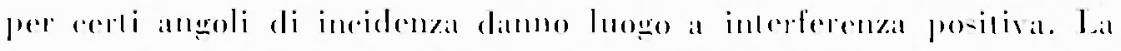

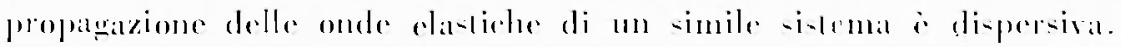
In un punto -mbeientemente di-lante i periodi predominamli salanmo fuelli aseociali rom valori stazonari della relorila di wruppo.

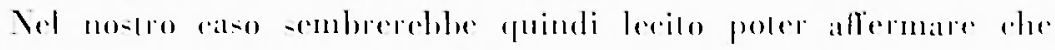
le onde e. f.... somo probahilmente onde superlediali del lipo residdello di Rayleigh. generale dall'urlo rontro la ro-la fe contro il londo?) delle onde longiludinali direlle e da quelle rillese piò rolu.

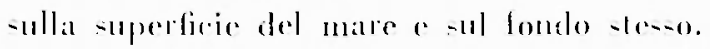

Queste onde hamno periodi molto prowimi. enrepondenti ai

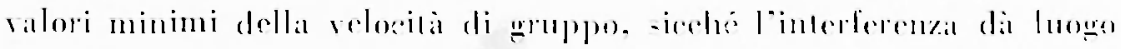

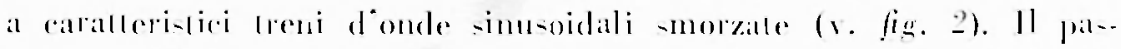

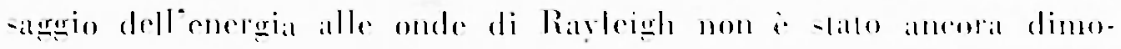

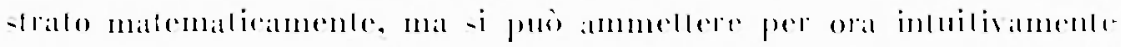

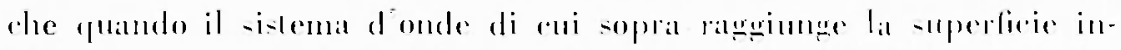

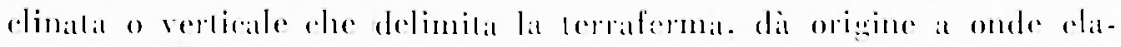

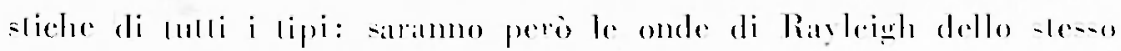

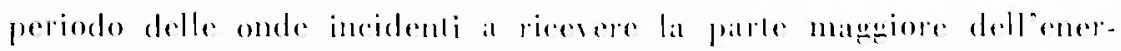
rial di furele.

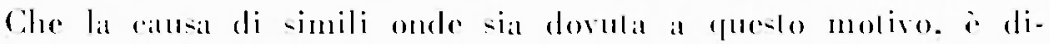
mostralo dal fallo rhe i rallalleristiri lreni donde simusoidali smor- 
zalle. di notewole ampierat. ci ri-contrano soltanlo per le explosioni in mare. mentere per quelle in lerra maneano quasi del lullo. Le notevoli ampiceze ora menzionale sono dovale al fallo che nell'alcepa lampieza dellonda longitudinale si mantiene pralicamente inallerata finclé le riflenioni sono lotali. e viene di poeo diminuila per valori dellangolo di incidenza pro-simi allangolo limite. Anche il raralleri-lieo comportamenlo delle dromoerone di que-le onde, men-

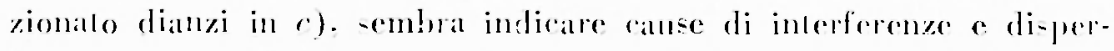
-ioni come guelle sopra menzionate, piullosto che mat differenziazio-

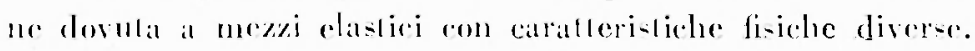

fienistenza di auesti treni d'onda nelle coplosioni in mare. qui chiaramente dimo-trata, is della masima importanza per lat disen.. cione sulle ratuse dei microsi-mi, in quanto remele ragione di wno dei fallori onemali piò importanli ma meno fareilmente spiegabili rela-

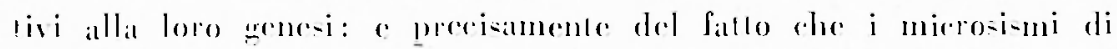

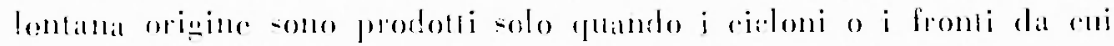

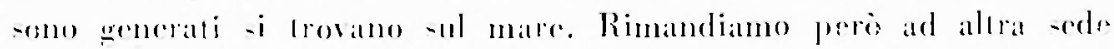

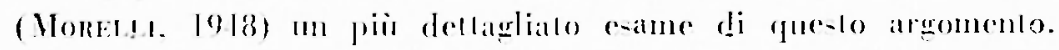

Istimbo Vazionale di Geofisira - Osecrvalorio di Trie-te, luplio 1919.

\section{RISSINTO}

Vellaprile-maggio 19.7 somo stali falli esplodere nel Golfo di rriesle alemini residuati bellici. appogsiale sul fondo del mare. appros-

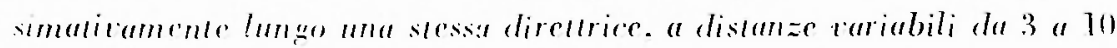
lim dalla Siazime Sismica. Poirhe le carallerishehe sismo-elasliche locali dello stralo superficiule crano finora scomosciule. is stato fallo

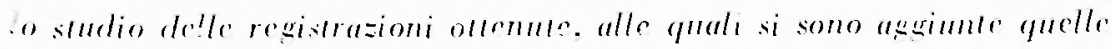

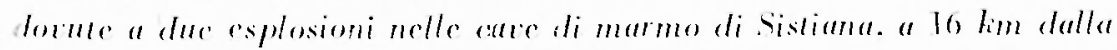

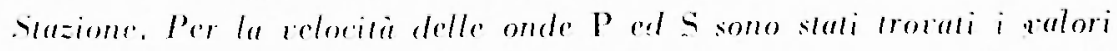
di 2630 a $1720 \mathrm{~m}$ sece rispellitamente. corrispondenti al rulore 0.15 per it coefficiente de Poisson. Sono stale quindi rostruite le dromo-

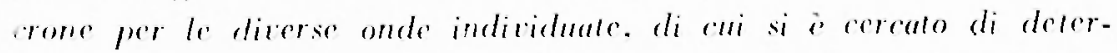
inimare te caralleristide. Parlicolarmente degne di menzione le noeroli ampiezas alle minori distamze delle onde longimdinali direlle 


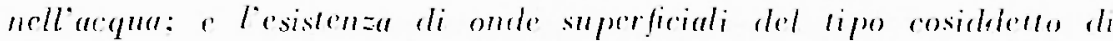
Rayleigh, generate secondo la teoria di Press ad Escing dallo per. turbazioni nel sistema acqua-suolo. Ouesta ronstalazione id fomblamentale imporlansa per la discusisome sulle ranse dei microsimmi.

\section{BIBI,IOCiRATII}

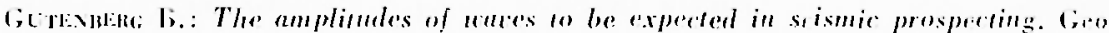
pluswil's. 1. 2. 1936.

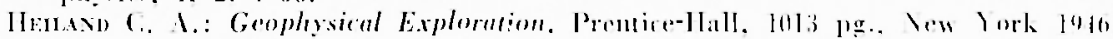

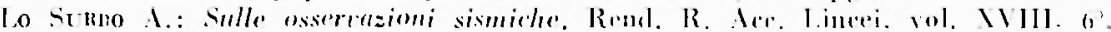
$\therefore 11,1909$.

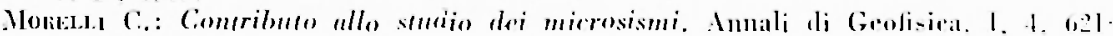
652. Romit 1918.

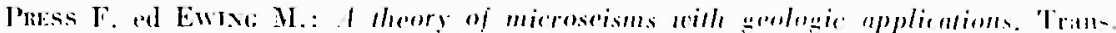

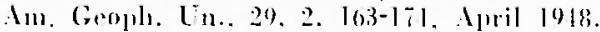

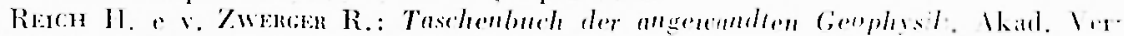

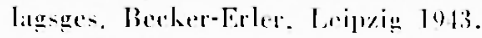

